\title{
Decoupling and iterative approaches to the control of discrete linear repetitive processes
}

\author{
B. Sulikowski · K. Gałkowski · E. Rogers • \\ D. H. Owens
}

Received: 31 March 2006 / Revised: 26 September 2006 /

Accepted: 5 December 2006 / Published online: 6 March 2007

(c) Springer Science+Business Media, LLC 2007

\begin{abstract}
This paper reports new results on the analysis and control of discrete linear repetitive processes which are a distinct class of $2 \mathrm{D}$ discrete linear systems of both systems theoretic and applications interest. In particular, we first propose an extension to the basic state-space model to include a coupling term previously neglected but which arises in some applications and then proceed to show how computationally efficient control laws can be designed for this new model.
\end{abstract}

Keywords Repetitive dynamics - Stability · 1D equivalent model · Dynamics decoupling · Iterative stabilization · LMIs

\section{Introduction}

The essential unique characteristic of a repetitive, or multipass, process is a series of sweeps, termed passes, through a set of dynamics defined over a fixed finite duration

B. Sulikowski $(\bowtie) \cdot$ K. Gałkowski

Institute of Control and Computation Engineering,

University of Zielona Góra, ul. Podgórna 50, 65-246 Zielona Góra, Poland

e-mail: b.sulikowski@issi.uz.zgora.pl

K. Gałkowski

e-mail: k.galkowski@issi.uz.zgora.pl

E. Rogers

School of Electronics and Computer Science,

University of Southampton, Southampton SO17 1BJ, UK

e-mail: etar@ecs.soton.ac.uk

D. H. Owens

Department of Automatic Control and Systems Engineering, University of Sheffield, Mappin Street, Sheffield S1 3JD, UK

e-mail: d.h.owens@sheffield.ac.uk 
known as the pass length. On each pass an output, termed the pass profile, is produced which acts as a forcing function on, and hence contributes to, the dynamics of the next pass profile. This, in turn, leads to the unique control problem in that the output sequence of pass profiles generated can contain oscillations that increase in amplitude in the pass-to-pass direction.

To introduce a formal definition, let $\alpha<+\infty$ denote the pass length (assumed constant). Then in a repetitive process the pass profile $y_{k}(p), 0 \leq p \leq \alpha-1$, generated on pass $k$ acts as a forcing function on, and hence contributes to, the dynamics of the next pass profile $y_{k+1}(p), 0 \leq p \leq \alpha-1, k \geq 0$.

Physical examples of repetitive processes include long-wall coal cutting and metal rolling operations (Edwards, 1974). Also in recent years applications have arisen where adopting a repetitive process setting for analysis has distinct advantages over alternatives. Examples of these so-called algorithmic applications include classes of iterative learning control (ILC) schemes (Owens, Amann, Rogers, \& French, 2000) and iterative algorithms for solving nonlinear dynamic optimal control problems based on the maximum principle (Roberts, 2002). In the case of ILC for the linear dynamics case, the stability theory for so-called differential and discrete linear repetitive processes can be used to undertake a rigorous stability/convergence theory for a powerful class of such algorithms. In particular, it makes explicit the link between error convergence and along the trial dynamics in a manner which is not possible using, for example, other 2D systems model structures, e.g. Kurek, \& Zaremba (1993).

Attempts to control these processes using standard (or 1D) systems theory/algorithms fail (except in a few very restrictive special cases) precisely because such an approach ignores the two features that define their inherent 2D systems structure. These are information propagation from pass-to-pass ( $k$ direction) and along a given pass ( $p$ direction) and resetting of the initial conditions before the start of each new pass. In which context, it is known (Owens, \& Rogers, 1999) that the structure of these alone can cause instability.

In seeking a rigorous foundation on which to develop a control theory for these processes, it is natural to attempt to exploit structural links which exist between, in particular, the class of so-called discrete linear repetitive processes and 2D linear systems described by the extensively studied Roesser (1975) or Fornasini and Marchesini (1978) state-space models. In fact, however, there are dynamics arising in repetitive processes which have no Roesser or Fornasini-Marchesini model interpretations. For example, it can happen that the previous pass profile is modified over its complete duration before the production of the next pass profile begins, e.g. in long-wall coal cutting where this so-called inter-pass smoothing is caused by the machines weight as it it brought to rest on the new cut floor profile before the start of the new pass of the coal face. Such dynamics cannot be included in a Roesser or Fornasini Marchesini model setting and the novel results in this paper include how a discrete model of such action can be accommodated in repetitive process analysis and control law design.

In common with a large range of other areas in systems theory, recent years has seen the emergence of Linear Matrix Inequality (LMI) based techniques in the analysis of very important sub-classes of linear repetitive processes (see, e.g., Galkowski, Rogers, Xu, Lam, \& Owens, 2002; Galkowski, Paszke, Sulikowski, Rogers, \& Owens, 2003). This has led to considerable success, especially in areas such as the structure and design of control laws and stability in the presence physically relevant types of uncertainty in the matrices of the defining state-space model, which have proved very difficult/impossible to handle using other analysis tools. In particular, use of LMIs has 
led, unlike other currently available alternatives, to the development of stability tests which also provide a basis for the design of physically meaningful control laws for both stability and performance. Here we show how such tools can be used to great effect in the control of the previously not considered repetitive process dynamics considered in this paper.

Throughout this paper $M>0(<0)$ denotes a real symmetric positive (negative) definite matrix.

\section{Background}

The state-space model of a so-called extended discrete linear repetitive process is described by the following state-space model over $0 \leq p \leq \alpha-1, k \geq 0$,

$$
\begin{aligned}
x_{k+1}(p+1) & =A x_{k+1}(p)+B u_{k+1}(p)+\sum_{j=0}^{\alpha-1} B_{j} y_{k}(j), \\
y_{k+1}(p) & =C x_{k+1}(p)+D u_{k+1}(p)+\sum_{j=0}^{\alpha-1} D_{j} y_{k}(j) .
\end{aligned}
$$

Here on pass $k, x_{k}(p)$ is the $n \times 1$ state vector, $y_{k}(p)$ is the $m \times 1$ pass profile vector, and $u_{k}(p)$ is the $r \times 1$ vector of control inputs.

To complete the process description, it is necessary to specify the boundary conditions, i.e. the state initial vector on each pass and the initial pass profile. Here no loss of generality arises from assuming $x_{k+1}(0)=d_{k+1}, k \geq 0$, where $d_{k+1}$ is an $m \times 1$ vector with known constant entries, and $y_{0}(p)=f(p)$, where $f(p)$ is an $m \times 1$ vector whose entries are known functions of $p$ over $0 \leq p \leq \alpha-1$. For ease of presentation, we will make no further reference to the boundary conditions in this paper.

Motivation for considering processes of the form (1) arises from applications where the current pass profile at any point along the pass is a function of more than one point on the previous pass. For example, in mining systems, the repetitive process dynamics arise from the fact that, as the current pass profile is being produced, the machine involved rests on the previous pass profile and once the end is reached it is returned to the starting position and then 'pushed over' to rest on the newly produced pass profile ready for the start of the next pass. Hence, it is clear that the complete previous pass profile in this case will make a significant contribution to the construction of the current pass profile at any point $p$ on the current pass. Consequently the resulting effects on the dynamics must be accounted for in any 'realistic' mathematical model.

The structure of (1) has no Roesser or Fornasini-Marchesini state-space model interpretation and hence the theory of such systems cannot be applied here. To deal with this case, we first derive new results for the previously considered discrete linear repetitive process state-space model and then extend them to this new model. In this previous model (Rogers, \& Owens, 1992), it was assumed that the current pass and state profile vector at any point was only directly influenced by the pass profile vector at the same point on the previous pass. Hence this model makes no allowance for the 'extra' dynamics described in the previous paragraph and the model structure of (1) is one possible means of including its effects. 
Suppose that for $p=0,1, \ldots, \alpha-1$

$$
B_{j}= \begin{cases}B_{0}, & j=p \\ 0, & j \neq p\end{cases}
$$

and also

$$
D_{j}= \begin{cases}D_{0}, & j=p, \\ 0, & j \neq p .\end{cases}
$$

Then in this case, the model of (1) reduces to that first introduced in Rogers, \& Owens (1992), i.e.

$$
\begin{aligned}
x_{k+1}(p+1) & =A x_{k+1}(p)+B u_{k+1}(p)+B y_{k}(p), \\
y_{k+1}(p) & =C x_{k+1}(p)+D u_{k+1}(p)+D_{0} y_{k}(p) .
\end{aligned}
$$

In this paper we will refer to this last model as a standard and the model of (1) as an extended discrete linear repetitive process respectively.

Consider the following partial ordering of two tuple integers

$$
\begin{array}{ll}
(i, j) \leq(k, p), & \text { if } i \leq k \text { and } j \leq p, \\
(i, j)=(k, p), & \text { if } i=k, j=p, \\
(i, j)<(k, p), & \text { if }(i, j) \leq(k, p) \text { and }(i, j) \neq(k, p) .
\end{array}
$$

Then the dynamics of the discrete linear repetitive processes considered in this paper can be visualized as evolving over the rectangle

$$
D_{e}:=\{(k, p): k \geq 0,0 \leq p \leq \alpha-1\} .
$$

Figure 1 shows the updating structure of the state and pass profile vectors in (1) (which clearly includes that of (4) as a special case).

Suppose also that it is required to ensure stability for a given example. Then in terms of control law actuation, information in the following sets has already been generated at point $p$ on pass $k$ and hence available for use in a control law

$$
\begin{aligned}
& X_{n c}=\left\{x_{k}(\tau): p<\tau \leq \alpha\right\} \cup\left\{x_{k^{\prime}}(p): 0 \leq p \leq \alpha, k^{\prime}<k\right\}, \\
& U_{n c}=\left\{u_{k}(\tau): p<\tau \leq \alpha-1\right\} \cup\left\{u_{k^{\prime}}(p): 0 \leq p \leq \alpha-1, k^{\prime}<k\right\}, \\
& Y_{n c}=\left\{y_{k}(\tau): p<\tau \leq \alpha-1\right\} \cup\left\{y_{k^{\prime}}(p): 0 \leq p \leq \alpha-1, k^{\prime}<k\right\} .
\end{aligned}
$$
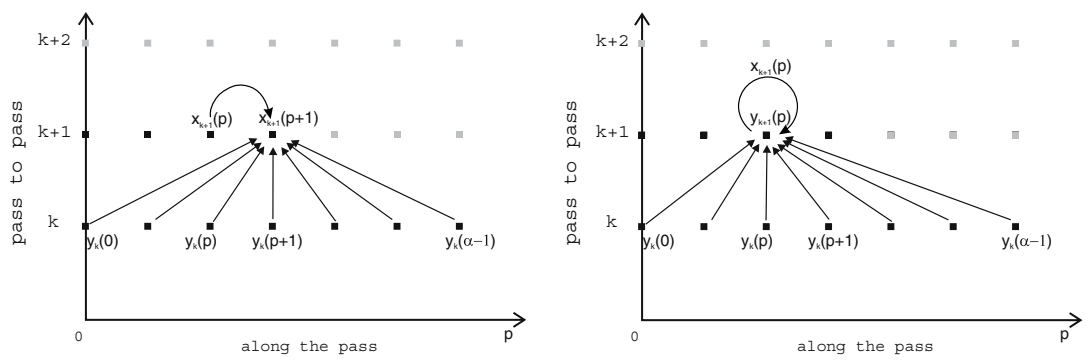

Fig. 1 Illustrating the state (left) and pass profile (right) vector updating structure in (1) 
For processes described by (4) it is (as noted previously in this paper) possible to solve some control related problems by exploiting their inherent 2D linear systems structure and, in effect, adapt tools/results first developed for 2D linear systems described by the extensively studied Roesser (1975) and Fornasini and Marchesini (1978) state-space models. In cases where this approach is not applicable, such as pass profile controllability (Galkowski, Rogers, \& Owens, 1998) which has no Roesser or Fornasini-Marchesini model equivalent, the 1D equivalent model (Galkowski, Rogers, \& Owens, 1998) has provided the analysis basis on which to characterize this property in terms of matrix rank tests and easily implemented stability tests respectively. Here we investigate the role of such an equivalent model for processes described by (1), where its construction is a straightforward extension of that for processes described by (4), and hence only the main steps are detailed here.

Set $l=k+1$ and $v_{l+1}(p)=y_{l}(p)$ in (1) and introduce the so-called global state, input and pass profile vectors (termed super-vectors here) of dimensions $n \alpha \times 1, r \alpha \times 1$ and $m \alpha \times 1$, respectively, as

$$
X(l):=\left[\begin{array}{c}
x_{l}(1) \\
x_{l}(2) \\
x_{l}(3) \\
\vdots \\
x_{l}(\alpha)
\end{array}\right], \quad U(l):=\left[\begin{array}{c}
u_{l}(0) \\
u_{l}(1) \\
u_{l}(2) \\
\vdots \\
u_{l}(\alpha-1)
\end{array}\right], \quad V(l):=\left[\begin{array}{c}
v_{l}(0) \\
v_{l}(1) \\
v_{l}(2) \\
\vdots \\
v_{l}(\alpha-1)
\end{array}\right] .
$$

Then the $1 \mathrm{D}$ equivalent model is

$$
\begin{aligned}
X(l) & =\Gamma_{\alpha} V(l)+\Sigma_{\alpha} U(l)+\Psi_{0_{\alpha}} d_{l}, \\
V(l+1) & =\Phi_{\alpha} V(l)+\Delta_{\alpha} U(l)+\Theta_{0_{\alpha}} d_{l},
\end{aligned}
$$

where

$$
\begin{aligned}
& \Psi_{0_{\alpha}}:=\left[\begin{array}{c}
A \\
A^{2} \\
A^{3} \\
\vdots \\
A^{\alpha}
\end{array}\right], \quad \Theta_{0_{\alpha}}:=\left[\begin{array}{c}
C \\
C A \\
C A^{2} \\
\vdots \\
C A^{\alpha-1}
\end{array}\right], \\
& \Sigma_{\alpha}:=\left[\begin{array}{ccccc}
B & 0 & 0 & \ldots & 0 \\
A B & B & 0 & \ldots & 0 \\
A^{2} B & A B & B & \ldots & 0 \\
\vdots & \vdots & \vdots & \ddots & \vdots \\
A^{\alpha-1} B & A^{\alpha-2} B & A^{\alpha-3} B & \ldots & B
\end{array}\right] \\
& \Gamma_{\alpha}:=\left[\begin{array}{ccccc}
B_{0} & B_{1} & B_{2} & \ldots & B_{\alpha-1} \\
B_{0}+A B_{0} & B_{1}+A B_{1} & B_{2}+A B_{2} & \ldots & B_{\alpha-1}+A B_{\alpha-1} \\
\sum_{i=0}^{2} A^{i} B_{0} & \sum_{i=0}^{2} A^{i} B_{1} & \sum_{i=0}^{2} A^{i} B_{2} & \ldots & \sum_{i=0}^{2} A^{i} B_{\alpha-1} \\
\sum_{i=0}^{3} A^{i} B_{0} & \sum_{i=0}^{3} A^{i} B_{1} & \sum_{i=0}^{3} A^{i} B_{2} & \ldots & \sum_{i=0}^{3} A^{i} B_{\alpha-1} \\
\vdots & \vdots & \vdots & \ddots & \vdots \\
\sum_{i=0}^{\alpha-1} A^{i} B_{0} & \sum_{i=0}^{\alpha-1} A^{i} B_{1} & \sum_{i=0}^{\alpha-1} A^{i} B_{2} & \ldots & \sum_{i=0}^{\alpha-1} A^{i} B_{\alpha-1}
\end{array}\right],
\end{aligned}
$$




$$
\Phi_{\alpha}:=\left[\begin{array}{ccccc}
D_{0} & D_{1} & D_{2} & \ldots & D_{\alpha-1} \\
C B_{0}+D_{0} & C B_{1}+D_{1} & C B_{2}+D_{2} & \ldots & C B_{\alpha-1}+D_{\alpha-1} \\
\sum_{i=0}^{1} C A^{i} B_{0}+D_{0} & \sum_{i=0}^{1} C A^{i} B_{1}+D_{1} & \sum_{i=0}^{1} C A^{i} B_{2}+D_{2} & \ldots & \sum_{i=0}^{1} C A^{i} B_{\alpha-1}+D_{\alpha-1} \\
\sum_{i=0}^{2} C A^{i} B_{0}+D_{0} & \sum_{i=0}^{2} C A^{i} B_{1}+D_{1} & \sum_{i=0}^{2} C A^{i} B_{2}+D_{2} & \ldots & \sum_{i=0}^{2} C A^{i} B_{\alpha-1}+D_{\alpha-1} \\
\vdots & \vdots & \vdots & \ddots & \vdots \\
\sum_{i=0}^{\alpha-2} C A^{i} B_{0}+D_{0} & \sum_{i=0}^{\alpha-2} C A^{i} B_{1}+D_{1} & \sum_{i=0}^{\alpha-2} C A^{i} B_{2}+D_{2} & \ldots & \sum_{i=0}^{\alpha-2} C A^{i} B_{\alpha-1}+D_{\alpha-1}
\end{array}\right],
$$

$$
\Delta_{\alpha}:=\left[\begin{array}{ccccc}
D & 0 & 0 & \ldots & 0 \\
C B & D & 0 & \ldots & 0 \\
C A B & C B & D & \ldots & 0 \\
\vdots & \vdots & \vdots & \ddots & \vdots \\
C A^{\alpha-2} B & C A^{\alpha-3} B & C A^{\alpha-4} B & \ldots & D
\end{array}\right]
$$

Next, we introduce the required parts of the stability theory for constant pass length linear repetitive processes and develop some new results on dynamic decoupling (defined in context in the next section).

\section{Stability and stabilization: the standard model case}

The stability theory (Rogers, \& Owens, 1992) for linear repetitive processes is based on an abstract model of the process dynamics in a Banach space setting and consists of two distinct concepts, termed asymptotic stability and stability along the pass, respectively. Recalling the unique control problem for these processes, asymptotic stability demands that bounded (defined in terms of the norm on the underlying signal space) input sequences (formed from control inputs and disturbances which enter on the current pass) produce bounded sequences of pass profiles over the, finite and fixed by definition, pass length. Also if this property holds then the sequence of pass profiles produced is guaranteed to converge in the pass-to-pass direction to a so-called steady or limit profile which in the case of the processes considered here are described by a $1 \mathrm{D}$ discrete linear systems state-space model.

For the remainder of this section, we consider only processes described by (4) since the extension to processes described by (1) is immediate (we will exploit dynamic decoupling as defined below in the next section). First note that asymptotic stability holds if, and only if, $r\left(D_{0}\right)<1$, where $r(\cdot)$ denotes the spectral radius of its argument. Also, with $D=0$ for simplicity, the $1 \mathrm{D}$ model describing the resulting limit profile dynamics has state matrix $A_{l P}:=A+B_{0}\left(I_{m}-D_{0}\right)^{-1} C$. Hence it is possible for a process to be asymptotically stable yet the resulting limit profile is 'unstable along the pass' in the $1 \mathrm{D}$ systems sense, i.e. $r\left(A_{l P}\right) \geq 1$. A simple example here is when $A=-0.5, B=1, B_{0}=-0.5+\beta, C=1, D=D_{0}=0$, where the real scalar $\beta$ is chosen such that $|\beta| \geq 1$.

This situation is due to the finite pass length (over which duration even an unstable $1 \mathrm{D}$ linear system can only produce a bounded output). Intuitively, therefore, it is to be expected that a stability definition which prevents this undesirable behaviour, termed stability along the pass, will place constraints on the matrix $A$ and, in particular, the 
requirement that $r(A)<1$, since this matrix describes the evolution of the dynamics along a pass. This is indeed true but, as the above example demonstrates, it still does not guarantee that unstable limit profile dynamics cannot occur. The extra condition required here is most appropriately expressed as the requirement that, assuming $\left\{A, B_{0}\right\}$ is controllable and $\{C, A\}$ observable, all eigenvalues of the transfer function matrix

$$
\mathcal{G}_{0}(z):=C\left(z I_{n}-A\right)^{-1} B_{0}+D_{0}
$$

have modulus strictly less than unity for all $|z|=1$.

It is also possible to give a physically based interpretation of these properties and the difference between them. Consider first asymptotic stability in the presence of no control input terms. Then at $p=0$ we have $y_{k}(0)=D_{0}^{k} y_{0}(0), k \geq 1$. Hence asymptotic stability can be interpreted as requiring that the sequence of pass initial profile vectors must not become unbounded with $k$. For stability along the pass consider for simplicity the single-input single-output case with zero state initial vector sequence and zero control input. Then the process dynamics can be written as $y_{k}(z)=\mathcal{G}_{0}^{k}(z) y_{0}(z)$ and hence we see that this property requires that each frequency component of the initial pass profile is attenuated from pass-to-pass at a geometric rate.

Suppose also that it is required to ensure stability for a given example. Then in terms of control law actuation, all the information in the sets of (5) is available for use. In implementation terms, however, there is clearly benefit to be achieved from using a control law which requires the minimal amount of information from these sets. Moreover, earlier work (Rogers, \& Owens, 1992) has shown that, except in a few very restrictive special cases, the control law used must be actuated by a combination of current pass information and 'feedforward' information from the previous pass to guarantee even stability along the pass with the control law applied. Note also here that in the ILC application area the previous pass output vector (or a trial in ILC terminology) is an obvious signal to use as feedforward action.

One control law with this structure is of the following form over $0 \leq p \leq \alpha-1, k \geq 0$

$$
u_{k+1}(p)=H_{x} x_{k+1}(p)+H_{y} y_{k}(p):=H\left[\begin{array}{c}
x_{k+1}(p) \\
y_{k}(p)
\end{array}\right],
$$

where $H_{x}$ and $H_{y}$ are appropriately dimensioned matrices to be designed. Note that (9) can be partitioned into two following control laws (depending on which part of model is to be influenced), i.e.

$$
u_{k+1}(p)=H_{y} y_{k}(p)+\hat{u}_{k+1}(p)
$$

or

$$
u_{k+1}(p)=H_{x} x_{k+1}(p)+\hat{u}_{k+1}(p),
$$

where now $\hat{u}_{k+1}(p)$ denotes an auxiliary control input which is available for the further use (if required).

In effect, the control law (9) uses feedback of the current state vector (which is assumed to be available for use) and 'feedforward' of the previous pass profile vector. Note that in repetitive processes the term 'feedforward' is used to describe the case where state or pass profile information from the previous pass (or passes) is used as (part of) the input to a control law applied on the current pass, i.e. to information which is propagated in the pass-to-pass $(k)$ direction. 
The stability properties of processes described by (4) can be compactly summarized in terms of the so-called augmented plant matrix

$$
\Upsilon:=\left[\begin{array}{cc}
A & B_{0} \\
C & D_{0}
\end{array}\right]
$$

and under the action of a control law of the form (10) this is mapped to

$$
\Upsilon_{c}:=\left[\begin{array}{cc}
A & B_{0}+B H_{y} \\
C & D_{0}+D H_{y}
\end{array}\right]
$$

and under (11) to

$$
\Upsilon_{c}:=\left[\begin{array}{ll}
A+B H_{x} & B_{0} \\
C+D H_{x} & D_{0}
\end{array}\right]
$$

(For the extended model (1) only the first of these mappings holds).

It now follows immediately that under the action of (10), the matrix $D_{0}$ governing asymptotic stability is mapped to $D_{0}+D H_{y}$ and hence the controlled process has this property if, and only if, the pair $\left\{D_{0}, D\right\}$ is controllable in normal 1D sense. Similarly, using (11), the matrix $A$ is mapped to $A+B H_{x}$ and it follows immediately that this control law can achieve the necessary condition for stability along the pass that $r\left(A+B H_{x}\right)<1$ if, and only if, the pair $\{A, B\}$ is controllable in the normal 1D sense. Note also that the application of (10) or (11) always maps the matrix $B_{0}$ or $C$, respectively, and hence the possibility of using it simplify the dynamics of the resulting controlled process by decoupling the pass state and pass profile updating equations in the manner detailed next.

Consider first (10) and suppose that it is possible to achieve

$$
\widetilde{B}_{0}:=B_{0}+B H_{y}=0
$$

by the suitable choice of $H_{y}$. Then the state dynamics on the current pass are completely decoupled from the previous pass profile and a simplified updating structure holds. In particular, given the pass state initial vector sequence $\left\{d_{k}\right\}_{k \geq 1}$ and the control input sequence to be applied, the state dynamics on each pass can be computed and then the corresponding pass profile sequence, i.e. in Figure 1 simplified to this case horizontal (state) and vertical (pass profile) updating become independent of each other. Also asymptotic stability holds provided we can also choose $H_{y}$ such that

$$
r\left(\widetilde{D}_{0}\right)<1, \quad \widetilde{D}_{0}:=D_{0}+D H_{y} .
$$

Suppose now that (11) is applied and it is also possible to achieve

$$
\widetilde{C}:=C+D H_{x}=0 .
$$

Then the state dynamics on the current pass are completely decoupled from the previous pass profile and a simplified updating structure then holds. In particular given the pass initial profile $y_{0}(p)$ and the control input sequence the pass profile sequence can be computed directly (and then the pass state vector sequence if required). Also it is possible that (11) can be designed such that

$$
r(\widetilde{A})<1, \quad \widetilde{A}:=A+B H_{x} .
$$

The following results now follow immediately from the above analysis and application of a well known 1D discrete linear system LMI controller design condition (see e.g. Galkowski et al., 2003). 
Theorem 1 Suppose that a control law of the form (11) is applied to a discrete linear repetitive process described by (4). Then (14) and (15) simultaneously hold if, and only if, there exist matrices $P_{x}>0, G_{x}$, and $N_{x}$ such that the following LMI holds

$$
\begin{gathered}
{\left[\begin{array}{cc}
-P_{x} & A G_{x}+B N_{x} \\
G_{x}^{T} A^{T}+N_{x}^{T} B^{T} & P_{x}-G_{x}-G_{x}^{T}
\end{array}\right]<0,} \\
C G_{x}+D N_{x}=0 .
\end{gathered}
$$

When this condition holds, $H_{x}$ can be computed as

$$
H_{x}=N_{x} G_{x}^{-1} .
$$

Theorem 2 Suppose that a control law of the form (10) is applied to a discrete linear repetitive process described by (4). Then (12) and (13) simultaneously hold if, and only if, there exist matrices $P_{y}>0, G_{y}$ and $N_{y}$ such that the following LMI holds

$$
\begin{gathered}
{\left[\begin{array}{cc}
-P_{y} & D_{0} G_{y}+D N_{y} \\
G_{y}^{T} D_{0}^{T}+N_{y}^{T} D^{T} & P_{y}-G_{y}-G_{y}^{T}
\end{array}\right]<0,} \\
B_{0} G_{y}+B N_{y}=0 .
\end{gathered}
$$

When this condition holds, $H_{y}$ can be computed as

$$
H_{y}=N_{y} G_{y}^{-1} \text {. }
$$

The following result concerning stability along the pass can also be stated.

Theorem 3 Suppose that a control law of the form (9) is applied to a discrete linear repetitive process described by (4). Then the resulting controlled process is stable along the pass if
(a) (16) holds,
(b) (18) holds,
(c) $\widetilde{C}=0$ or $\widetilde{B}_{0}=0$.

Proof The inequalities (16) and (18) are equivalent to $r(\widetilde{A})<1$ and $r\left(\widetilde{D}_{0}\right)<1$, respectively (Sulikowski, Galkowski, Rogers, \& Owens, 2005). Now consider the controlled process version of (8), i.e.

$$
\widetilde{\mathcal{G}_{0}}(z):=\widetilde{C}\left(z I_{n}-\widetilde{A}\right)^{-1} \widetilde{B}_{0}+\widetilde{D}_{0} .
$$

Then clearly $\widetilde{C}=0$ or $\widetilde{B}_{0}=0$ guarantees

$$
r\left(\widetilde{\mathcal{G}}_{0}(z)\right)=r\left(\widetilde{D}_{0}\right)<1 .
$$

The analysis above is, of course, subject to several strong limitations which may hinder its applicability. First, the two matrix equations in this last result must be solved and the existence of a solution requires that

$$
\operatorname{rank}(D)=\operatorname{rank}\left(\left[D, C G_{x}\right]\right)
$$

and

$$
\operatorname{rank}(B)=\operatorname{rank}\left(\left[B, B_{0} G_{y}\right]\right) .
$$


The point here is that the decision matrices $G_{x}$ and $G_{y}$ in the LMIs are also the part of the conditions for the existence of solutions which can cause problems. If no solutions of (17) and (19) exist, then one possibility is to attempt approximate decoupling by minimizing a norm applied to $C G_{x}+D N_{x}$ and $B_{0} G_{y}+B N_{y}$.

This decoupling based approach can also be applied to the model of (1) but stability along the pass is a much more involved question. Moreover, design for asymptotic stability could include the need to work with (potentially) very large dimensioned matrices. We show in the next section, however, that this problem can be avoided by adopting a different design strategy. Finally, note that uncertainty in the model matrices in the above design can also be treated using known techniques. In particular, if there is uncertainty in $C$ and $D$ for the first case and $B_{0}$ and $B$ for the second then analysis based on uncertainty models with a known norm bounded structure (see, for example, Galkowski et al., 2003) or polytopic form (Cichy, Galkowski, Kummert, \& Rogers, 2005) extends directly - the equality constraints (17) and (19), respectively, do not change their forms and (16) and (18) respectively need only be rewritten in the form of the considered uncertainty structure.

\section{Stability and stabilization analysis: the extended model case}

Consider first asymptotic stability of the extended model where the situation is clearly much more complicated than for the standard model. There is, however, a route forward based on the fact that asymptotic stability in this case is equivalent to requiring $r\left(\Phi_{\alpha}\right)<1$ in the 1D equivalent model (which can be proved as in Rogers, Galkowski, Gramacki, Gramacki, \& Owens, 2002 for the case of dynamic boundary conditions.)

This is a very powerful result and the only real difficulty with it is the (potentially) very large dimensions of the matrix involved. Note also that major numerical errors can occur during the construction of the $1 \mathrm{D}$ equivalent model due to the need to form powers of the matrix $A$, especially when $r(A)>1$.

Numerical difficulties could also arise in terms of control law design to ensure asymptotic stability. To explain this point, note that for processes described by (4), asymptotic stability can be achieved (here we assume that this is possible) under a control law of the form (9) (with $H_{x}=0$ for simplicity) by choosing the matrix $H_{y}$ such that $r\left(D_{0}+D H_{y}\right)<1$. In the case of processes described by (1), however, this is not possible and here we show that an alternative is to employ the $1 \mathrm{D}$ equivalent model and seek to design a control law of the form

$$
U(l)=K V(l)
$$

such that $r\left(\Phi_{\alpha}+\Delta_{\alpha} K\right)<1$. This control law is actuated by all points along the previous pass where as (9) is only single point actuated. Given that in the model of (1) it is all points along the previous pass which contribute to the dynamics at a single point on the current pass (plus the fact that asymptotic stability deals with information in the pass-to-pass $(k)$ direction) it is to be expected that a control law with single point actuation will be relatively weak in this case.

As the first step in developing an efficient design algorithm for (20) to ensure asymptotic stability, we proceed via an LMI interpretation arising from the fact that $r\left(\Phi_{\alpha}\right)<1$ is equivalent to the requirement that $\exists P>0$ such that

黑 Springer 


$$
\left[\begin{array}{cc}
-P & \Phi_{\alpha}^{T} P \\
P \Phi_{\alpha} & -P
\end{array}\right]<0
$$

Hence we have the following necessary and sufficient condition for asymptotic stability under the action of the control law (20).

Theorem 4 Suppose that the 1D equivalent model (6) is used to design a control law of the form (20) for a discrete linear repetitive process described by (1). The controlled process is asymptotically stable if, and only if, $\exists$ matrices $Q>0$ and $L$ such that the following LMI holds

$$
\left[\begin{array}{cc}
-Q & Q \Phi_{\alpha}^{T}+L^{T} \Delta_{\alpha}^{T} \\
\Phi_{\alpha} Q+\Delta_{\alpha} L & -Q
\end{array}\right]<0 .
$$

Also if this condition holds then $K$ in (20) can be computed as

$$
K=L Q^{-1} \text {. }
$$

Proof The controlled process is asymptotically stable (using the Lyapunov stability inequality for 1D discrete linear systems with state matrix $\Phi_{\alpha}$, input matrix $\Delta_{\alpha}$ and state feedback matrix $K$ ) if, and only if,

$$
\left(\Phi_{\alpha}+\Delta_{\alpha} K\right)^{T} P\left(\Phi_{\alpha}+\Delta_{\alpha} K\right)-P<0,
$$

where $P>0$. Applying the Schur's complement formula for matrices and pre- and post-multiplying the result as detailed next

$$
\left[\begin{array}{ll}
I & 0 \\
0 & P
\end{array}\right]\left[\begin{array}{cc}
-P & \left(\Phi_{\alpha}+\Delta_{\alpha} K\right)^{T} \\
\Phi_{\alpha}+\Delta_{\alpha} K & -P^{-1}
\end{array}\right]\left[\begin{array}{ll}
I & 0 \\
0 & P
\end{array}\right]<0
$$

yields

$$
\left[\begin{array}{cc}
-P & \left(\Phi_{\alpha}+\Delta_{\alpha} K\right)^{T} P \\
P\left(\Phi_{\alpha}+\Delta_{\alpha} K\right) & -P
\end{array}\right]<0 .
$$

Next, set $Q=P^{-1}$ and pre- and post-multiply (26) by the diagonal matrix $\operatorname{diag}\{Q, Q\}$ to obtain

$$
\left[\begin{array}{cc}
-Q & Q\left(\Phi_{\alpha}+\Delta_{\alpha} K\right)^{T} \\
\left(\Phi_{\alpha}+\Delta_{\alpha} K\right) Q & -Q
\end{array}\right]<0 .
$$

Use of (23) now yields the LMI of (22) and the proof is complete.

A difficulty in applying this result may arise since $Q$ is a LMI decision matrix and simultaneously is used to compute the control law matrix $K$. The next result is better in this respect and is based on the 1D case as in Peaucelle, Arzelier, Bachelier, \& Bernussou (2000).

Theorem 5 Suppose that the 1D equivalent model (6) is used to design a control law of the form (20) for a discrete linear repetitive process described by (1). Then the controlled process is asymptotically stable if, and only if, $\exists$ matrices $P>0$ and $G, L$ such that the following LMI holds

$$
\left[\begin{array}{cc}
-P & \Phi_{\alpha} G+\Delta_{\alpha} L \\
G^{T} \Phi_{\alpha}^{T}+L^{T} \Delta_{\alpha}^{T} & P-G-G^{T}
\end{array}\right]<0 .
$$


Also if this condition holds, a stabilizing $K$ is given by

$$
K=L G^{-1} \text {. }
$$

Proof For necessity assume that $G=Q$ and then (28) reduces to (22). For sufficiency, left multiply (28) by $\left[I \mid \Phi_{\alpha}+\Delta_{\alpha} L G^{-1}\right]$ (note that $G$ is invertible since $G+G^{T}>0$ ) and right multiply the result by the transpose of this matrix to obtain (24).

Both of the design methods given above require the solution of possibly very large dimensioned LMIs which is well known to be difficult to do using modern solvers. Next, we show that this problem can be overcome in some cases of direct interest here by exploiting the decoupling results of the previous section. In particular, suppose that the current pass state vector is decoupled from the pass profile updating equation in (1) using the control law $u_{k+1}(p)=H_{x} x_{k+1}(p)+\hat{u}_{k+1}(p)$, such that $C+D H_{x}=0$ (where $\hat{u}_{k+1}(p)$ is an auxiliary current pass control input vector). (Note that in the design algorithm which follows $C$ and $D$ must be exactly known and the extension to robustness analysis as discussed at the end of the previous section can only be applied here when the uncertainty is not present in these matrices.)

The resulting controlled process state-space model after the control law has been designed is

$$
\begin{aligned}
x_{k+1}(p+1) & =\left(A+B H_{x}\right) x_{k+1}(p)+B \hat{u}_{k+1}(p)+\sum_{j=0}^{\alpha-1} B_{j} y_{k}(j), \\
y_{k+1}(p) & =D \hat{u}_{k+1}(p)+\sum_{j=0}^{\alpha-1} D_{j} y_{k}(j)
\end{aligned}
$$

and in the $1 \mathrm{D}$ equivalent model we have that

$$
\Phi_{\alpha}=\left[\begin{array}{ccccc}
D_{0} & D_{1} & D_{2} & \ldots & D_{\alpha-1} \\
D_{0} & D_{1} & D_{2} & \ldots & D_{\alpha-1} \\
\vdots & \vdots & \vdots & \ddots & \vdots \\
D_{0} & D_{1} & D_{2} & \ldots & D_{\alpha-1}
\end{array}\right], \Delta_{\alpha}=\left[\begin{array}{ccccc}
D & 0 & 0 & \ldots & 0 \\
0 & D & 0 & \ldots & 0 \\
\vdots & \vdots & \vdots & \ddots & \vdots \\
0 & 0 & 0 & \ldots & D
\end{array}\right] .
$$

Now we have the following easily proved result which, in effect, is a computationally more efficient test for asymptotic stability.

Lemma 1 The matrix $\Phi_{\alpha}$ of (31) has $(\alpha-1) m$ zero eigenvalues and the remaining $m$ are those of the matrix $\sum_{j=0}^{\alpha-1} D_{j}$.

The form of $\Phi_{\alpha}$ here can also be exploited in the design of a control law of the form

$$
\hat{U}(l)=K V(l),
$$

where $\hat{U}(l)$ is the $1 \mathrm{D}$ equivalent model super-vector corresponding to $\hat{u}_{k+1}(p)$, to ensure asymptotic stability of the original process. In particular, suppose that the structure of the control law matrix is taken to be 


$$
K=\left[\begin{array}{ccccc}
K_{0} & K_{1} & K_{2} & \ldots & K_{\alpha-1} \\
K_{0} & K_{1} & K_{2} & \ldots & K_{\alpha-1} \\
\vdots & \vdots & \vdots & \vdots & \vdots \\
K_{0} & K_{1} & K_{2} & \ldots & K_{\alpha-1}
\end{array}\right]
$$

which yields a $1 \mathrm{D}$ equivalent model with state matrix

$$
\tilde{\Phi}_{\alpha}=\Phi_{\alpha}+\Delta_{\alpha} K
$$

Then we have the following result.

Theorem 6 Suppose that a control law of the form $u_{k+1}(p)=H_{x} x_{k+1}(p)+\hat{u}_{k+1}(p)$ is applied to a discrete linear repetitive process described by (1) and has been designed such that (30) holds. Suppose also that (30) is replaced by its 1D equivalent model (6) which is then used to design a control law of the form (32) (i.e. $\hat{U}(l)=K V(l)$ ) applied with a $K$ of the form (33). Then the resulting controlled process is asymptotically stable if, and only if, $\exists$ matrices $P>0, G, L_{j}, j=0,1, \ldots, \alpha-1$ such that the following $L M I$ holds

$$
\left[\begin{array}{cc}
-P & \sum_{j=0}^{\alpha-1}\left(D_{j} G+D L_{j}\right) \\
\sum_{j=0}^{\alpha-1}\left(G^{T} D_{j}^{T}+L_{j}^{T} D^{T}\right) & P-G-G^{T}
\end{array}\right]<0 .
$$

Also if this condition holds, block entry $K_{j}$ in the control law matrix $K$ of (33) is given by

$$
K_{j}=L_{j} G^{-1}, \forall j=0,1, \ldots, \alpha-1
$$

Proof First, set $\mathcal{D}=\sum_{j=0}^{\alpha-1} D_{j}, \mathcal{K}=\sum_{j=0}^{\alpha-1} K_{j}$ and $\mathcal{L}=\sum_{j=0}^{\alpha-1} L_{j}$. Then the resulting controlled process is asymptotically stable if, and only if,

$$
(\mathcal{D}+D \mathcal{K})^{T} W(\mathcal{D}+D \mathcal{K})-W<0,
$$

where $W>0$. Next make an obvious application of the Schur's complement formula and pre- and post-multiply the result by $\left[\begin{array}{ll}I & 0 \\ 0 & W\end{array}\right]$ to obtain

$$
\left[\begin{array}{cc}
-W & (\mathcal{D}+D \mathcal{K})^{T} W \\
W(\mathcal{D}+D \mathcal{K}) & -W
\end{array}\right]<0 .
$$

Now set $P=W^{-1}$ and pre- and post-multiply (38) by the $\operatorname{diagonal}$ matrix $\operatorname{diag}\{P, P\}$ to obtain

$$
\left[\begin{array}{cc}
-P & P(\mathcal{D}+D \mathcal{K})^{T} \\
(\mathcal{D}+D \mathcal{K}) P & -P
\end{array}\right]<0
$$

or, with $\mathcal{K}=\mathcal{L} P^{-1}$,

$$
\left[\begin{array}{cc}
-P & P \mathcal{D}^{T}+\mathcal{L}^{T} D^{T} \\
\mathcal{D} P+D \mathcal{L} & -P
\end{array}\right]<0 .
$$


Now we have to establish that (40) is equivalent to (35), where this last condition can be replaced by

$$
\left[\begin{array}{cc}
-P & \mathcal{D} G+D \mathcal{L} \\
G^{T} \mathcal{D}^{T}+\mathcal{L}^{T} D^{T} & P-G-G^{T}
\end{array}\right]<0 .
$$

To show necessity, assume $G=P$ and note that (41) becomes (40) since the LMI is symmetric. For sufficiency, left multiply (41) by $\left[I \mid \mathcal{D}+D \mathcal{L} G^{-1}\right]$ (note that $G$ is invertible since $G+G^{T}>0$ ) and right multiply the result by its transpose to obtain (37). Next note that

$$
\mathcal{K}=\mathcal{L} G^{-1}=\left(\sum_{j=0}^{\alpha-1} L_{j}\right) G^{-1}=\sum_{j=0}^{\alpha-1}\left(L_{j} G^{-1}\right)=\sum_{j=0}^{\alpha-1} K_{j}
$$

and (35) ensures that the control law matrix $\mathcal{K}$ is such that

$$
r\left(\left(\sum_{j=1}^{\alpha-1} D_{j}\right)+D \mathcal{K}\right)<1
$$

which, on applying the result of Lemma 1, is equivalent to $r\left(\tilde{\Phi}_{\alpha}\right)<1$. This completes the proof.

Remark 1 Note here that the LMI to be solved in this last result is, in general, of much less dimension than that of Theorem 5. In particular, we have to compute an $m \alpha \times m \alpha$ decision matrix in the LMI of Theorem 5 and in that of Theorem 6 an $m \times m$ matrix, where most often $\alpha$ (the pass length) is much greater than $m$ (the number of entries in the pass profile vector).

A simplified form of the above result arises when it is assumed that

$$
K_{i j}=\hat{K}, \quad i, j=0,1, \ldots, \alpha-1 .
$$

Also if this condition holds then it is easy to conclude that $\mathcal{K}=\alpha \hat{K}$, and from (42) $\mathcal{L}$ of (35) satisfies $\mathcal{L}=\alpha \hat{L}$. Hence we can then use

$$
K=\left[\begin{array}{ccccc}
\hat{K} & \hat{K} & \hat{K} & \ldots & \hat{K} \\
\hat{K} & \hat{K} & \hat{K} & \ldots & \hat{K} \\
\vdots & \vdots & \vdots & \vdots & \vdots \\
\hat{K} & \hat{K} & \hat{K} & \ldots & \hat{K}
\end{array}\right]
$$

In summary, therefore, we have developed a procedure for control law design to achieve asymptotic stability of processes described by (1). The first step is to use preliminary control action to decouple the current pass state vector from the pass profile updating equation (which, however, is not always possible). This step does not reduce the dimension of, in particular, the matrix $\Phi_{\alpha}$ but crucially greatly simplifies its spectrum. It is this fact which simplifies the final control law design.

Consider now stability along the pass. Then here we need to consider the case when $\alpha \rightarrow \infty$ and it is clear that the stability along the pass analysis of the previous section is not applicable here. Suppose, however, that preliminary control action has been applied to convert the process dynamics to the form (30). Also introduce 
$\Phi_{\infty}:=\lim _{\alpha \rightarrow \infty} \Phi_{\alpha}$. Then it can be argued directly from the abstract model based theory that stability along the pass holds if, and only if, $r\left(\Phi_{\infty}\right)<1$. This in turn, is equivalent to $r\left(\sum_{j=0}^{\infty} D_{j}\right)<1$ which is more convenient for numerical evaluation.

\section{Successive stabilization}

The analysis of the previous section has produced a systematic method of ensuring asymptotic stability using the $1 \mathrm{D}$ equivalent model. A consequence of this, however, is that the inherent 2D linear systems structure of these processes has been subsumed into the 1D model and in some cases of interest it will be beneficial to retain this structure. Also to meet the decoupling specifications of the previous analysis, extra conditions are necessary which will not always hold. Conversely, direct use of Theorem 4 or 5 could lead in some cases to computations involving matrices of extremely high dimensions and hence the strong possibility of overflow and underflow errors in intermediate steps which can completely destroy the numerical reliability of the calculation process. (The problem here arises from the multiplication of the input, pass profile and state vector dimensions by the value of the pass length during the construction of the $1 \mathrm{D}$ equivalent model.)

As an alternative, we now develop the so-called successive stabilization approach. In effect, this first replaces the potentially huge dimensioned problem with one of less dimensions and then iteratively improves it to the full pass length subject to the requirement that the outcome of each stage must result in a state-space model which has the structure of a discrete linear repetitive process (this is not guaranteed in the cases where Theorem 4 or 5 can be applied).

To improve the iterative solution algorithm, the preliminary step of stabilizing the matrix $A$ in the $1 \mathrm{D}$ discrete linear systems sense when $r(A) \geq 1$ can lead to a reduction in the number of iterations needed to obtain the required result. Note also that successive stabilization is not an alternative solution in cases when Theorem 4 or 5 does not hold. Rather the aim is to obtain an efficient and numerically reliable solution in the case when at least one of these results holds but is not computationally feasible with a high degree of accuracy.

To proceed, suppose that the control law matrix $K$ applicable in (20) is assumed to be of the form (33), where this ensures that the controlled process retains the structure of (1). Hence $K$ can be treated as a set of 2D controllers $K_{i}, i=0, \ldots, \alpha-1$, which influence the dynamics from pass-to-pass.

The structure of (33) for $K$ can be achieved by setting

$$
G=\left[\begin{array}{cccc}
G_{0} & 0 & \ldots & 0 \\
0 & G_{1} & \ldots & 0 \\
\vdots & \vdots & \vdots & \vdots \\
0 & 0 & \ldots & G_{\alpha-1}
\end{array}\right]
$$

and

$$
L=\left[\begin{array}{ccccc}
L_{0} & L_{1} & L_{2} & \ldots & L_{\alpha-1} \\
L_{0} & L_{1} & L_{2} & \ldots & L_{\alpha-1} \\
\vdots & \vdots & \vdots & \vdots & \vdots \\
L_{0} & L_{1} & L_{2} & \ldots & L_{\alpha-1}
\end{array}\right]
$$


in the condition of Theorem 5. Then $K_{0}=L_{0} G_{0}^{-1}, K_{1}=L_{1} G_{1}^{-1}, \ldots, K_{\alpha-1}=$ $L_{\alpha-1} G_{\alpha-1}^{-1}$, and also $\widetilde{\Phi}_{\alpha}$ can be written in the form $\widetilde{\Phi}_{\alpha}=\left[\widetilde{\Phi}_{i j}\right]$, where

$$
\begin{aligned}
\widetilde{\Phi}_{i j} & =\left\{\begin{array}{l}
D_{j}+D K_{j}, \quad i=1, \quad j=0,1, \ldots, \alpha-1, \\
D_{j}+D K_{j}+\sum_{t=0}^{i-1}\left(C A^{t} B_{j}+C A^{t} B K_{j}\right), \quad i=2,3, \ldots, \alpha, \quad j=0,1, \ldots, \alpha-1
\end{array}\right. \\
& =\left\{\begin{array}{l}
\widehat{D}_{j}, \quad i=1, \\
\widehat{D_{j}}+\sum_{t=0}^{i-1}\left(C A^{t} \widehat{B}_{j}\right), \quad i=2,3, \ldots, \alpha-1 .
\end{array}\right.
\end{aligned}
$$

Hence, it follows immediately that the controlled process with the matrix $K$ given by (33) has a state-space model of the form (1) with $\widehat{A}=A, \widehat{B}=B, \widehat{C}=C, \widehat{D}=D$ and

$$
\widehat{B}_{i}=B_{i}+B K_{i}, \quad \widehat{D}_{i}=D_{i}+D K_{i}, \quad i=0,1, \ldots, \alpha-1 .
$$

The basic idea of so-called successive stabilization is that we first make the process asymptotically stable over a short pass length and then subsequently augment this design by increasing the pass length by using only control action which preserves the original repetitive process state-space model structure. This approach to design also avoids the need to work with matrices with very large dimensions.

The design procedure is as follows.

Step 1 Choose an initial pass length $\alpha_{1}<\alpha$, and $h$ the number of points added in each iteration such that there exists a natural number $q$ satisfying $\alpha-\alpha_{1}=q h$. Set an iteration counter $v=1$.

Step 2 Construct $\Phi_{\alpha_{1}}$ using (7) with $\alpha=\alpha_{1}$ and check if $r\left(\Phi_{\alpha_{1}}\right)<1$. If yes, go to Step 5.

Step 3 Use (28) and (29) to compute the appropriate control law matrices $K_{0}, K_{1}, \ldots$, $K_{\alpha_{1}-1}$ for the partial pass of the length $\alpha_{1}$. Set $K_{\alpha_{1}}, K_{\alpha_{1}+1}, \ldots, K_{\alpha-1}=0$ and calculate the full modified system matrix $\tilde{\Phi}_{\alpha}$ of (34).

Step 4 Check if $r\left(\tilde{\Phi}_{\alpha}\right)<1$, and if this is true terminate the procedure. If not, go to the next step.

Step 5 Check if $\alpha_{1}+h>\alpha$ holds. If yes, terminate the procedure since asymptotic stability for the controlled process cannot be achieved by this method. If no, increase $v$ by 1 , set $\alpha_{1}:=\alpha_{1}+h$ and return to Step 2 .

Remark 2 Note that above algorithm is always at least as effective as stabilization based on direct use of the matrix $\Phi_{\alpha}$. To see this, set $\alpha_{1}=\alpha$ in the first step. Also if the initial stabilization problem size is $\alpha_{1}<\alpha$, and it is impossible to achieve stabilization for such a chosen pass length, then executing Step 5 finally gives stabilization based on $\Phi_{\alpha}$.

Remark 3 For sufficiently large $\alpha$ and small $\alpha_{1}$ and $h$ the evaluation of the above algorithm can take a very long time but overall we have found that it compares very favourably with solving the stabilization problem with the full $\Phi_{\alpha}$.

Next, we give numerical examples to illustrate the results in this paper, the first for decoupling and the second successive stabilization. 


\section{Examples}

Example 1 Consider the particular case of (1) when $\alpha=20$ and

$$
\begin{aligned}
& A=\left[\begin{array}{ccc}
1.99 & -0.96 & 0.0 \\
0.0 & 0.0 & -1.87 \\
-2.36 & -2.82 & -0.32
\end{array}\right] \\
& B=\left[\begin{array}{ccc}
0.0 & 1.54 & 2.13 \\
2.24 & 0.0 & -1.43 \\
0.92 & -2.03 & 1.18
\end{array}\right] \\
& \mathbf{B}:=\left[B_{0}\left|B_{1}\right| \ldots \mid B_{19}\right] \\
& =\left[\begin{array}{cc|cc|cc|cc|cc}
-1.44 & -0.96 & -2.85 & 0.0 & 0.0 & 1.33 & 1.47 & 0.0 & 0.88 & -0.87 \\
0.0 & -2.54 & 0.0 & -0.71 & 1.86 & -1.87 & 0.0 & 0.0 & 2.81 & 2.20 \\
0.0 & 2.07 & 0.0 & 0.10 & 2.62 & 0.0 & 2.40 & 3.0 & 0.53 & -0.29
\end{array} \mid\right.
\end{aligned}
$$

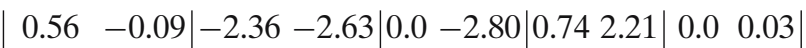

$$
\begin{aligned}
& \begin{array}{ll|ll|ll|ll|ll|}
-1.88 & -0.33 & 0.0 & 0.0 & 0.0 & 1.92 & 1.77 & 0.0 & 2.58 & 0.14
\end{array} \\
& \begin{array}{ll|lll|ll|l|l|l|l|}
1.33 & 0.0 & 1.0 & 0.0 & 0.0 & -1.27 & 2.83 & 0.67 & 1.04 & 0.51
\end{array} \\
& \begin{array}{|ll|ll|ll|ll|ll|}
0.03 & 0.0 & 0.0 & 1.03 & -0.37 & -2.15 & 0.82 & 2.95 & -1.59 & -0.96
\end{array} \mid \\
& \begin{array}{lll|ll|ll|ll|ll|}
0.0 & 0.50 & 0.0 & 0.0 & 1.24 & 0.56 & 0.0 & 0.0 & 2.02 & -2.84
\end{array} \\
& \begin{array}{ll|llll|ll|ll}
0.0 & -2.96 & 0.0 & -2.22 & 0.0 & 0.0 & 0.0 & 1.08 & -0.84 & -0.32
\end{array} \\
& \left.\mid \begin{array}{cc|cc|cc|cc|cc}
-2.02 & -0.47 & -1.45 & 2.09 & 0.0 & 0.68 & 0.25 & 0.07 & 2.60 & 0.0 \\
1.94 & 0.76 & 0.60 & 0.0 & -0.81 & 0.0 & 2.87 & -1.85 & -0.15 & -0.13 \\
-2.19 & 0.0 & -1.18 & -1.67 & -0.98 & -2.63 & 0.0 & 0.0 & 0.99 & 0.0
\end{array}\right] \text {, } \\
& C=\left[\begin{array}{ccc}
1.38 & 1.69 & -2.21 \\
0.0 & 0.0 & 1.62
\end{array}\right] \\
& D=\left[\begin{array}{ccc}
-0.46 & -2.89 & 2.34 \\
0.0 & -0.18 & -2.12
\end{array}\right] \\
& \mathbf{D}:=\left[D_{0}\left|D_{1}\right| \ldots \mid D_{19}\right] \\
& =\left[\begin{array}{cc|cc|cc|cc|cc}
0.24 & 0.0 & 0.0 & -1.67 & 0.0 & 0.49 & 0.0 & -0.90 & -1.46 & 1.68 \\
0.94 & 1.17 & -1.54 & 1.91 & 0.0 & -1.74 & -0.77 & -0.78 & 0.0 & -1.66
\end{array} \mid\right. \\
& \left|\begin{array}{cc|cc|cc|cc|cc}
-1.92 & 0.05 & -0.6 & 0.0 & 0.0 & 1.49 & -0.16 & 0.72 & 0.0 & 1.13 \\
0.95 & 0.34 & 0.0 & -1.79 & 0.0 & 0.0 & -0.68 & -1.43 & -1.25 & 1.87
\end{array}\right| \\
& \begin{array}{|lll|l|ll|ll|ll|}
0.0 & 0.0 & 1.13 & -1.43 & 0.0 & 0.0 & 0.0 & -0.91 & 0.06 & 0.17
\end{array}
\end{aligned}
$$

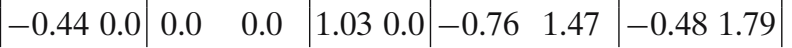




$$
\left.\mid \begin{array}{cc|cc|cc|cc|cc}
0.0 & -1.15 & 0.0 & 0.62 & -0.04 & 0.20 & 0.0 & 1.45 & 0.72 & 1.49 \\
-0.83 & -1.73 & -0.77 & 0.0 & -0.92 & -1.75 & -1.06 & 0.0 & 0.0 & 0.0
\end{array}\right] .
$$

This example is asymptotically unstable since $r\left(\Phi_{\alpha}\right)=6.9248 \times 10^{7}$. Applying Theorem 1 in this case yields the following matrices

$$
\begin{gathered}
P_{x}=\left[\begin{array}{ccc}
1609.2545 & -3472.7949 & -3717.4772 \\
-3472.7949 & 8289.9193 & 8846.9029 \\
-3717.4772 & 8846.9029 & 9450.1232
\end{array}\right], \\
G_{x}=\left[\begin{array}{ccc}
970.4892 & -1856.2049 & -1998.9717 \\
-2065.2941 & 4755.6368 & 5082.2434 \\
-2206.7096 & 5048.4788 & 5413.2067
\end{array}\right], \\
N_{x}=\left[\begin{array}{ccc}
-3378.4267 & 6543.5446 & 7105.4660 \\
108.1323 & 108.6375 & 90.05 \\
-1695.4403 & 3848.5759 & 4128.8612
\end{array}\right]
\end{gathered}
$$

and the decoupling control law matrix $H_{x}$ is given by

$$
H_{x}=\left[\begin{array}{ccc}
-2.9399 & -3.7553 & 3.7526 \\
0.8846 & 1.1064 & -0.6955 \\
-0.0751 & -0.0939 & 0.8232
\end{array}\right]
$$

Application of Theorem 6 now yields

$$
\begin{aligned}
& P=\left[\begin{array}{cc}
407022367.2187 & 0.0 \\
0.0 & 407022367.2187
\end{array}\right] \\
& G=\left[\begin{array}{cc}
407022367.2187 & 0.0 \\
0.0 & 407022367.2187
\end{array}\right]
\end{aligned}
$$

and if (38) and (39) are employed then

$$
\hat{L}=\left[\begin{array}{cc}
-8203055.3853 & 1106302.6858 \\
-60008273.8591 & 5489886.5988 \\
-58070224.4638 & -22833155.3180
\end{array}\right]
$$

and

$$
\hat{K}=\left[\begin{array}{cc}
-0.0202 & 0.0027 \\
-0.1474 & 0.0135 \\
-0.1427 & -0.0561
\end{array}\right]
$$

Finally, it is easy to see that the resulting controlled process is asymptotically stable as required.

The following example illustrates the successive stabilization method in the case when it is required to compute with much larger matrices (due to the value of the pass length).

Example 2 Consider the particular case of (1) when $\alpha=100$ and

$$
A=\left[\begin{array}{cc}
0.47 & 0.66 \\
0.66 & -0.11
\end{array}\right], \quad B=\left[\begin{array}{l}
0.58 \\
0.68
\end{array}\right], \quad C=[0.160], \quad D=[0.14]
$$




$$
\begin{aligned}
& \mathbf{B}:=\left[B_{0}\left|B_{1}\right| \ldots \mid B_{99}\right]
\end{aligned}
$$

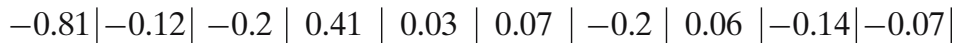

$$
\begin{aligned}
& \begin{array}{c|c|c|c|c|c|c|c|c|c|c}
0.05 & 0.04 & 0.22 & 0.4 & 0.4 & -0.35 & -0.02 & -0.2 & 0.24 & -0.03 & \ldots \\
\hline-0.14 & -0.15 & -0.14 & -0.15 & -0.03 & -0.06 & -0.01 & -0.01 & -0.07 & 0.02 &
\end{array} \\
& \begin{array}{llllllllllllll}
0.02 & -0.02 & 0.02 & 0.05 & -0.05 & 0 & 0.08 & 0 & 0.02 & 0.03 & \ldots \\
\hline
\end{array} \\
& \begin{array}{lll|l|l|l|l|l|l|l|l|}
\hline 0.02 & -0.07 & 0.09 & -0.07 & -0.06 & 0.04 & -0.05 & -0.02 & -0.07 & -0.05 \\
\hline
\end{array} \\
& \begin{array}{c|c|c|c|c|c|c|c|c|c|c}
0.06 & 0.07 & 0.03 & 0.02 & 0.08 & 0.06 & 0 & -0.03 & -0.02 & -0.05 & \ldots \\
\hline-0.02 & 0.04 & -0.04 & 0.06 & 0.05 & -0.01 & 0.06 & -0.02 & -0.02 & -0.05 &
\end{array} \\
& \begin{array}{c|c|c|c|c|c|c|c|c|c|c}
0.02 & -0.01 & -0.02 & -0.01 & -0.04 & 0.05 & 0 & -0.03 & 0.04 & -0.03 & \ldots \\
\hline-0.05 & -0.04 & -0.04 & -0.03 & 0.01 & 0.04 & 0.04 & 0.02 & 0.01 & 0.03 &
\end{array} \\
& =\begin{array}{c|c|c|c|c|c|c|c|c|c|c}
0.05 & 0.04 & -0.03 & 0.03 & 0.02 & 0.01 & -0.02 & -0.03 & 0.01 & -0.02 & \ldots \\
\hline-0.01 & 0.04 & 0.02 & 0.02 & -0.04 & 0.01 & -0.03 & 0.03 & 0.01 & 0.03 & \ldots
\end{array} \\
& \begin{array}{l|l|l|l|l|l|l|l|l|l|l}
-0.02 & 0.02 & -0.03 & -0.03 & 0 & -0.02 & -0.02 & 0.03 & 0.03 & 0.03 & \ldots \\
\hline
\end{array} \\
& \begin{array}{c|c|c|c|c|c|c|c|c|c|c}
\hline 0.03 & 0.02 & -0.01 & -0.02 & 0 & -0.02 & 0 & 0.02 & 0.01 & -0.03 & \\
0 & -0.02 & 0.01 & -0.02 & -0.03 & 0 & -0.01 & -0.02 & 0.03 & 0.02 & \ldots \\
\hline 0 & 0.01 & 0.03 & -0.03 & 0.01 & 0.01 & 0.01 & 0 & 0.02 & -0.02 & \\
0 & 0.01 & 0.03 & -0.01 & -0.03 & 0 & -0.02 & -0.01 & -0.01 & 0.02 & \ldots \\
\hline 0 & 0.01 & -0.02 & -0.01 & 0.01 & 0 & 0.01 & -0.01 & -0.02 & 0.01 & \\
-0.01 & 0.01 & -0.02 & 0 & 0.02 & -0.03 & -0.01 & 0.02 & -0.01 & -0.02 & \ldots \\
\hline-0.01 & 0.01 & -0.01 & -0.01 & 0.01 & -0.02 & -0.01 & -0.02 & 0 & -0.01 & \\
0.02 & -0.02 & -0.02 & 0.01 & 0 & 0.02 & 0 & 0.01 & 0.01 & 0.02 &
\end{array} \\
& \mathbf{D}:=\left[D_{0}\left|D_{1}\right| \ldots \mid D_{99}\right] \\
& \begin{array}{c|c|c|c|c|c|c|c|c|c|c}
-0.41 & -0.27 & -0.6 & 0.53 & 0.37 & -0.03 & 0.04 & 0 & 0.24 & -0.21 & \ldots \\
\hline-0.1 & -0.06 & -0.13 & 0.08 & 0.04 & -0.08 & -0.01 & -0.06 & -0.11 & -0.08 & \ldots \\
\hline 0.01 & 0.08 & 0.08 & 0.04 & -0.05 & 0 & 0.05 & 0.03 & -0.08 & -0.03 & \ldots \\
\hline-0.05 & -0.04 & -0.05 & -0.06 & -0.01 & -0.03 & -0.05 & -0.01 & -0.02 & 0.04 & \ldots \\
\hline-0.05 & 0.05 & 0.05 & 0.05 & 0.03 & -0.04 & -0.02 & 0.02 & -0.04 & 0.04 & \ldots \\
\hline 0 & 0.04 & -0.01 & 0.03 & -0.04 & 0.01 & -0.01 & 0.01 & -0.02 & 0.02 & \ldots \\
\hline-0.03 & -0.02 & 0 & 0.03 & -0.03 & 0 & -0.02 & 0.02 & 0 & 0.02 & \ldots \\
\hline-0.02 & 0.02 & -0.03 & 0.01 & 0.03 & -0.01 & -0.01 & 0 & 0.01 & -0.01 & \ldots \\
\hline 0.02 & 0.02 & 0 & 0 & -0.02 & 0 & -0.02 & 0 & -0.01 & 0.01 & \ldots \\
\hline 0.02 & -0.01 & -0.01 & 0 & -0.01 & 0.02 & -0.01 & 0 & -0.01 & 0 &
\end{array}
\end{aligned}
$$

Here $r\left(\Phi_{\alpha}\right)=1.4876$ and this process is asymptotically unstable. Moreover, attempts to design a control law for asymptotic stability by the direct route failed because the required LMI cannot be solved due to its large dimension.

To commence the successive stabilization algorithm, set $\alpha_{1}=10$ and $h=10$. Then Step 1 does not provide the required stabilizing control law but Step 2 is successful and yields the stabilizing control law matrices as

$$
K^{2}=\left[K_{1}^{2} K_{2}^{2}\right],
$$

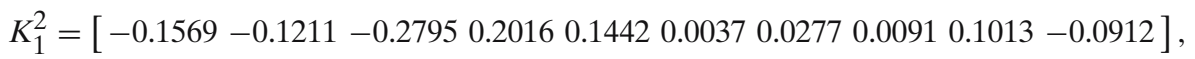

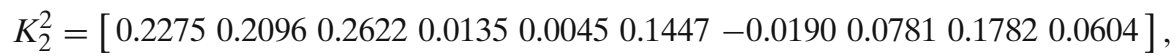


where the superscript here denotes the iteration number. To apply the above control law for the whole $\Phi_{\alpha}$ extend it by $0_{1 \times 80}$ (i.e. $K=\left[\begin{array}{ll}K^{2} & 0\end{array}\right]$ ) and hence $\tilde{\Phi}_{\alpha}=\Phi_{\alpha}+\Delta K$. The spectral radius of subsequent matrices $\tilde{\Phi}_{\alpha}$ (the matrix to which $\Phi_{\alpha}$ is mapped to under the control action) during these iterations are given in the following table

\begin{tabular}{lr}
\hline Iteration number & $r\left(\tilde{\Phi}_{\alpha}\right)$ \\
\hline 0 & 1.4876 \\
1 & 1.6738 \\
2 & 0.8379 \\
\hline
\end{tabular}

Here asymptotic stability for the controlled process is achieved after two iterations. Note also that when the direct route was attempted, the LMI involved was found to be unsolvable numerically.

Figures 2 and 3 below show the free evolution (i.e. $U(l)=0, l=0,1, \ldots$ ) without and with the control law applied respectively and boundary conditions $x_{k}(0)=$ $\left[\begin{array}{c}1.8 \\ -1.08\end{array}\right], k=1,2, \ldots$ and $y_{0}(p)=0.1,0 \leq p \leq 99$.

\section{Conclusions}

Control law design for discrete linear repetitive processes can involve the need to compute with extremely large dimensioned matrices and one of the major contributions of this paper is on the development of methods to avoid this problem. The first set of results relate to the use of preliminary control law action to completely decouple the effects of the current pas state and/or the previous pass profile vectors from the onward evolution of the process dynamics, with consequent simplifications in terms of control law design.



Fig. 2 The uncontrolled pass profile sequence 




Fig. 3 The controlled process pass profile sequence

The second set of results relate to the control of a new model for discrete linear repetitive processes to include a term missing from the currently used model but which could arise in applications, i.e. it is needed to capture the essential dynamics to be controlled. As a result, control law design algorithms based on, e.g., LMI methods may well encounter serious numerical difficulties and, moreover, the decoupling results developed in the first part of this paper only work in very restrictive special cases. To overcome these difficulties, a new stabilization procedure has been developed where the control law is designed by an iterative procedure and as the examples (and, in particular, Example 2) given, illustrate it can be applied to cases where very large dimensioned matrices arise. Further development, e.g. by using parallel/array processing, should allow for even larger examples well outside the range of most examples encountered and hence give a general and reliable design methodology. Finally, note that some of the LMI based results here are sufficient but not necessary and hence there is a degree of conservativeness associated with them. However, they are design algorithms which can be computed (as the examples here demonstrate) and, given the absence/computational intractability of necessary and sufficient conditions based on 2D polynomials, provide an applications oriented route forward.

\section{References}

Cichy, B., Galkowski, K., Kummert, A., \& Rogers, E. (2005). Control of discrete linear repetitive processes with variable parameter uncertainty. In Proceedings of the second international conference on informatics in control, automation and robotics-ICINCO 2005, Barcelona, Spain, vol. 3, pp. 37-42, CDROM.

Edwards, J. B. (1974). Stability problems in the control of multipass processes. Proceedings of The Institution of Electrical Engineers, 121(11), 1425-1431.

Fornasini, E., \& Marchesini, G. (1978). Doubly-indexed dynamical systems: state-space models and structural properties. Mathematical Systems Theory, 12, 59-72. 
Galkowski, K., Paszke, W., Sulikowski, B., Rogers, E., \& Owens, D. H. (2003). LMI based stability analysis and robust controller design for discrete linear repetitive processes. International Journal of Robust and Nonlinear Control, 13(13), 1195-1211.

Galkowski, K., Rogers, E., \& Owens, D. H. (1998). Matrix rank based conditions for reachability/controllability of discrete linear repetitive processes. Linear Algebra and its Applications, 275-276, 201-224.

Galkowski, K., Rogers, E., Xu, S., Lam, J., \& Owens, D. H. (2002). LMIs - a fundamental tool in analysis and controller design for discrete linear repetitive processes. IEEE Transactions on Circuits and Systems I: Fundamental Theory and Applications, 49(6), 768-778.

Kurek, J. E., \& Zaremba, M. B. (1993). Iterative learning control synthesis based on 2-D system theory. IEEE Transactions on Automatic Control, 38, 121-125

Owens, D. H., \& Rogers, E. (1999). Stability analysis for a class of 2D continuous-discrete linear systems with dynamic boundary conditions. Systems and Control Letters, 37, 55-60.

Owens, D. H., Amann, N., Rogers, E., \& French, M. (2000) Analysis of linear iterative learning control schemes - a 2D systems/repetitive processes approach. Multidimensional Systems and Signal Processing, 11, 125-177

Peaucelle, D., Arzelier, D., Bachelier, O., \& Bernussou, J. (2000). A new robust D-stability condition for polytopic uncertainty. Systems and Control Letters, 40, 21-30.

Roberts, P. D. (2002). Two-dimensional analysis of an iterative nonlinear optimal control algorithm. IEEE Transactions on Circuits and Systems I: Fundamental Theory and Applications, 49(6), 872878.

Roesser, R. P. (1975). A discrete state-space model for linear image processing. IEEE Transactions on Automatic Control, 20,1-10.

Rogers, E., \& Owens, D. H. (1992). Stability analysis for linear repetitive processes. Springer-Verlag Lecture Notes in Control and Information Sciences Series, Vol. 175, Berlin.

Rogers, E., Galkowski, K., Gramacki, A., Gramacki, J., \& Owens, D. H. (2002). Stability and controllability of a class of 2D linear systems with dynamic boundary conditions. IEEE Transactions on Circuits and Systems I: Fundamental Theory and Applications, 49(2), 181-195

Sulikowski, B., Galkowski, K., Rogers, E., \& Owens, D. H. (2005). Control and disturbance rejection for discrete linear repetitive processes. Multidimensional Systems and Signal Processing, 16(2), 199-216.

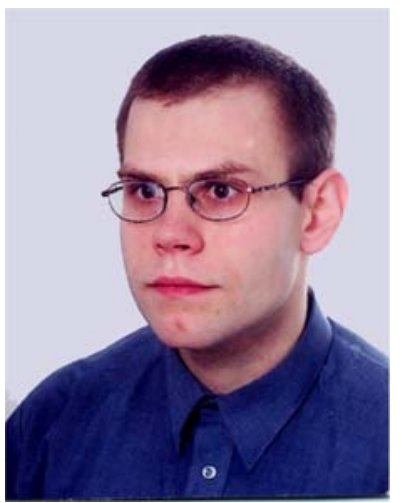

Bartlomiej Sulikowski received the Ph.D. degree from the University of Zielona Gora, Poland in February, 2006 and the M.Sc. degree from the same university in June, 2000. He has completed his Ph.D under the supervision of Prof. Krzysztof Galkowski (public defense had a place on February 1st, 2006). Since January, 1999 he had been working as a technical engineer at Institute of Robotics and Software Engineering, Technical University of Zielona Gora. After receiving the M.Sc. degree in Electrical Engineering with option for specialization in Computational Systems Engineering (final score: Excellent), in September, 2000 he has been appointed as a Assistant at Institute of Control and Computation Engineering, University of Zielona Gora (new name of Institute of Robotics and Software Engineering, Technical University of Zielona Gora). Since May, 2006 he has been appointed as a Assistant Professor at the same institute. His research interests are on multidimensional $(\mathrm{nD})$ systems, linear repetitive processes, and the application of numerical methods to control problems for $\mathrm{nD}$ systems. He spent one month training in the University of Southampton, UK, in 2003 funded by a grant from the British Council (no. WAR/342/11). He had been the member of a team carrying out the grant funded by the National Committee for Scientific Research no. 2T11A 020 (Polish Ministry of Science) in years 2001-2003. He was also a member of the research team of bilateral cooperation joint project between France and Poland POLONIUM, No. 4941/R2003/2004, (with INRIA Rocquencourt) and he has been a member of a team to handle the grant funded by the National Committee for Scientific Research no. 3T11A 008 (Polish Ministry of Science) in years 2004-2006. For his activity, he was twice awarded the President of University of Zielona Góra Teamwork First and Second Class Awards (2003 and 2006, respectively) . His publication record counts over 25 papers in the world-known journals and conference proceedings (e.g. journals: Automatica, MDSSP; 
conferences: ACC, ECC, CCA, MTNS, NDS). Recently he published the book entitled "Computational aspects in analysis and synthesis of repetitive processes" (University of Zielona Góra Press, 2006) summarizing his research so far. He has been a reviewer for known international conferences (e.g. CDC-ECC 2005, IFAC 2005). Furthermore, has was a member of the organizing committee of the 4th International Workshop on Multidimensional (nD) Systems, Wuppertal, Germany (July 1114, 2005). Mr. Sulikowski's teaching interests include Computer Networks (he's the certified CISCO CCNA instructor), Numerical Methods and Basics of Computer Programming.



Krzysztof Gałkowski received the M.S., Ph.D. and Habilitation (D.Sc.) degrees in electronics/automatic control from Technical University of Wrocław, Poland in 1972, 1977 and 1994 respectively. In October 1996 he joined the Technical University of Zielona Góra (now the University of Zielona Góra), Poland where he holds the professor position, and he is a visitng professor in the School of Electronics and Computer Science, University of Southampton, UK. In 2002, he was awarded the degree "Professor of Technical Science" the highest scientific degree in Poland. He is an associate editor of Int. J. of Multidimensional Systems and Signal Processing, International Journal of Control and Int. J. of Applied Mathematics and Computer Science. He has co-organised 4 International Workshops NDS 1998 Lagov, Poland, NDS 2000 - Czocha Castle - Poland, the third as a Symposium within MTNS 2002 in Notre Dame IN, US, and the last one NDS 2005 in Wuppertal, Germany. In 2007 NDS 2007 is organized in Aveiro, Portugal. His research interests include multidimensional (nD) systems and repetitve processes - theory and applications, control and related numerical and symbolic algebra methods. He is an author/editor of four monographs/books and about 60 papers in peer reiviewed journals and over 100 in the proceedings of international conferences. He leads an active group of young researchers in the $\mathrm{nD}$ systems area with strong international co-operation with the Universities of Southampton/for which he is awarded the official position of Visiting Professor at The School of Electronics and Computer Science/and Sheffield UK; the universities of Wuppertal, ErlangenNurnberg, Magdeburg, Germany; University of Hong Kong, University of Poitiers, IRIA, Sophia Antipolis, France. He is spending (spent) the academic year 2006-07 (2004/05) as a guest professor in The University of Wuppertal, Germany. In 2004, he obtained a Siemens Award for his research contributions.



Professor David Owens has been the Head of the Department of Automatic Control and Systems Engineering since 1999 and was Dean of Engineering at Sheffield University in the period 2002-2006. He was the Convener of the Deans in the period 2005-2006. Prior to this he was Head of the School of Engineering and Computer Science at the University of Exeter where he also acted as Chairman of Exeter Enterprises Ltd (the University Technology Transfer Company). Following 4 years with the UKAEA, he has over 34 years involvement in academic life with experience of departmental teaching, administration and research and high-level University committee service in three UK Universities (Sheffield, Strathclyde and Exeter Universities). He has acted as external examiner for both undergraduate programmes at universities including Glasgow, Bath, Sheffield, Coventry, De Montfort, Salford, Loughborough, KUTKM and UPM-Malaysia. He has had a strong involvement with the Engineering and related Professions having served on many Committees and the Council of the Institution of Electrical Engineers (IET) and currently serves on its Sector Panel for C\&I. He is a member and has chaired the Informatics and Control Group of the Institution of Mechanical Engineers and has been a member of both the IMechE Research and Standards Board and Technology Strategy Committee. He was the elected Chairman of the United Kingdom Automatic 
Control Council from 1999-2002 representing the interests of the UK Professional Institutions within IFAC. He has served on the Health and Safety Commission's Nuclear Safety Advisory Committee (1995-2006) and has been an independent member of British Energy's Training Standards and Accreditation Board. He is an Editor of an Institute of Mathematics and Its Applications International Journal, is an Associate Editor of several other journals and regularly contributes to international activities through conference organisation, seminars and invited lectures and committees of the International Federation of Automatic Control (IFAC).



Eric Rogers was born in 1956 near Dungannon in Northern Ireland. He read Mechanical Engineering as an undergraduate in The Queen's University of Belfast UK and was awarded his $\mathrm{PhD}$ degree by The University of Sheffield UK for a Thesis in the area of Multidimensional Systems Theory. He has been with the University of Southampton UK since 1990 where he is currently Professor of Control Systems Theory and Design in The School of Electronics and Computer Science. Prior to moving to Southampton, he held lectureship posts in The Queen's University of Belfast UK (1984-1987) and the University of Strathclyde UK (1988-1990). His current major research interests include multidimensional systems theory and applications, with particular emphasis on Behavioral Systems Theory Approaches and systems with Repetitive Dynamics, Iterative Learning Control, Flow Control, and Active Control of Microvibrations. He is currently the editor of The International Journal of Control, an associate editor of Multidimensional Systems and Signal Processing, and joint editor of The Taylor and Francis research book series on Systems and Control. In addition, he has served extensively on IEEE, IFAC and IEE technical committees and acted as a consultant to numerous companies and government agencies in UK and abroad. 